\title{
Economía Social y Solidaria: la necesidad de una pedagogía solidaria para contrarrestar la ambigüedad y la incertidumbre económica
}

\author{
Paulo P. de Albuquerque \\ Universidad Federal do Rio Grande do Sul - Brasil
}

\author{
SOCIAL AND SOLIDARITY ECONOMY:THE NEED FOR SOLIDARITY \\ PEDAGOGY TO COUNTER ECONOMIC AND ECONOMIC \\ UNCERTAINTY
}

\section{Resumen}

El artículo reflexiona sobre las ventajas de la asociación/cooperación y de la organización solidaria en un mundo complejo que exige retomar los presupuestos del paradigma asociativo y de la necesidad de una pedagogía política para contrarrestar al escenario societario de ambigüedad e incertidumbre de la contemporaneidad; pensar solidaridad implica en cambiar de matriz analítica de la modernidad y señala que la no-banalización de los principios de la participación y del compromiso necesitan se concretizar en un conjunto de medidas políticas que buscan la democratización y socializar los saberes que de hecho pasa por una pedagogía solidária.

Palabras clave

Praxis pedagógica; Solidaridad; Educación en la Economía social y solidaria

Códigos JEL: B55, P13, J54, A11, A12 Y A14

\section{Abstract}

This paper reflects on the advantages of the association/ cooperation and the solidarity organization in a complex world. It requires reestablishing the presuppositions of cooperative paradigm and the necessity of a political pedagogy to contradict the society stage of ambiguity and contemporaneity uncertainty because to think solidarity implies changing the analytical base of modernity. It points that the non-trivialization of participation and commitment principles need to be concretized in a set of policy measures that search for the democratization and socialization of knowledge that per pass solidarity pedagogy.

\section{Keywords}

Pedagogy; Solidarity; Social Economy Education

JEL codes: B55, P13, J54, A11, A12 Y A14

Fecha de recepción del original: 17 de julio de 2018; versión definitiva: 20 de diciembre de 2019.

Paulo P de Albuquerque, Facultad de Educación de la Universidad Federal do Rio Grande do Sul - Brasil

Tel. 005551 34072161; E-mail: albuquerque.paulo@ufrgs.br; albuquerque.paulo@gmail.com.

ORCID ID: 0000-003-49486761 


\section{Economía Social y Solidaria: la necesidad de una pedagogía solidaria para contrarrestar la ambigüedad y la incertidumbre económica}

Paulo P. de Albuquerque

Universidad Federal do Rio Grande do Sul - Brasil

Un verdadero viaje de descubrimiento no es buscar nuevas tierras, sino obtener otra mirada. Marcel Proust
1. Introducción

Toda reflexión depende de ciertas suposiciones en torno a la naturaleza de la realidad. Si aceptamos eso, las suposiciones funcionan como hipótesis; en caso contrario funcionan como creencias, aunque ambas traducen un modo de analizar la realidad a partir de paradigmas (y eso es peligroso).

Analizar un supuesto real o una posición es resultado de una percepción, de un impulso de evaluar otras lecturas y proponer otros modos de percibir una realidad que puedan hacernos dudar de nuestras propias interpretaciones y de nuestro habitual curso de acción. Ese impulso es mucho más que una autoafirmación, es proponer al interlocutor que hay otros modos de comprender la realidad, que la realidad admite la pluralidad de análisis.

Este trabajo tiene la intención (y la pretensión) de articular argumentos para pensar a partir de una pedagogía política, incluso desde una política pedagógica para contrarrestar la ambigüedad y la incertidumbre económica por la solidaridad de la economía social y solidaria. Puede ser que la argumentación tenga tintes de precariedad en su propuesta, pero en este caso la precariedad se utiliza como categoría analítica que no descalifica la propuesta de conversación que el texto busca.

Pensar las ventajas de la asociación/cooperación y de la organización solidaria en un mundo complejo exige retomar los presupuestos del paradigma cooperativista y resignificarlo frente al nuevo escenario societario lleno de ambigüedades e incertidumbres propias de la contemporaneidad, porque pensar la solidaridad implicar abandonar la matriz analítica de la modernidad iluminista. Tal posicionamiento se hace necesario, principalmente porque la matriz ideológica y epistémica del siglo pasado continúa fundamentándose en los imperativos inapelables de un pensamiento único (la racionalidad ins- trumental) de que el fenómeno social debe estar subordinado a los dictámenes del económico, lo que se ha transformado no solamente en invocación ritual, sino en la única lógica social posible de explicar la modernidad, desarrollo y democracia: la vida. Esto ha generado una lectura del mundo equivoca$\mathrm{da}$, porque es fundamentalmente normativa. Normativa, en la medida que condiciona el modo en que vemos las cosas; esta lógica simplista presenta el fenómeno social y económico como equivalentes y la sociedad compleja como resultado de acuerdos fundados en lógicas hegemónicas que traducen intereses idénticos de algunos actores sociales: gobierno y empresarios

De esta manera, usar la economía social y solidaria como estrategia para contrarrestar la ambigüedad y las incertidumbres de la economía supone dos cuestiones que actúan como hipótesis de trabajo:

1. ¿Existe en Latinoamérica un conjunto de fuerzas sociales que comparten intereses, puntos de vista y otras formas de lucha contra las consecuencias y los efectos perversos de una política económica?

2. ¿El supuesto carácter aletargado, poco creativo o relegado de la sociedad civil en los países del Sur es verdadero porque existe el boicot de las expresiones de la sociedad civil y de los movimientos sociales que impide a las organizaciones contrarrestar las incertidumbres de una economía que se presenta como globalizada?

Estas cuestiones pueden parecer ingenuas, a la luz de la cantidad cada vez mayor de organizaciones sociales y cooperativas que surgen en los países del tercer mundo, pero la virulencia de la realidad y del fundamentalismo económico parece indicar un vacío organizativo y un lamentable mimetismo estratégico y táctico que estas organizaciones suelen padecer. En ese sentido, la movilización social y la democratización pro- 
movidas por el tercer sector ${ }^{1}$ parecen romper con la visión tradicional y liberal del "quehacer" político que permite combinar igualdad política con desigualdad social y económica, porque se propone relacionar la pluralidad y la especificidad de los intereses sociales (autonomía) con la integración realizada por el Estado en otro nivel que no sea la mera subordinación.

En efecto, la construcción de nuevos espacios políticos y económicos deriva no solamente de la separación de sociedad civil y Estado, también de la ruptura de los aspectos formales de la democracia. En otras palabras, la separación de sociedad civil y Estado es la primera etapa de un proceso de "ciudadanía emancipada", donde la autonomización creciente de los actores sociales frente a las instituciones políticas traduce un modo diferenciado de representarlos. Por supuesto, para comprender o explicar las estrategias asociativas y solidarias en América latina se debe considerar la diversidad de escenarios que se presentan, pero también los diferentes significados de la organización solidaria que es la cooperativa.

2. Prolegómenos o por qué los significados precisan ser "resignificados"

Vivimos un período paradójico, por un lado, los procesos de transformación del trabajo redefinidos por el principio de la acción neoliberal han dado lugar a nuevas prácticas en el mercado de trabajo, siendo su efecto más visible la concentración de la renta que genera el individualismo, las desigualdades sociales y el desempleo (Quijano, 2005; Rosso, 1996; Singer, 2002; Santos y Rodríguez, 2005). Por otro lado, se ha producido un efecto de signo contrario que se manifiesta en la construcción de estrategias sociales que tienen en el asociacionismo y en la cooperación los fundamentos para generar otras formas de relaciones sociales más solidarias.

Este inicio de siglo se caracteriza por la inestabilidad y por la incertidumbre; pues a la crisis económica y social se añade también una crisis política. Se puede hablar con certeza de que la actual crisis no es una mera crisis sectorial, localizada o geográfica, sino una crisis plural y generalizada.

En palabras de Rafael Correa en el Foro Social Mundial (Belém do Pará - Brasil edición 2009): “No se trata solamente de una crisis económica, es el resultado de la codicia, del egoísmo y del individualismo erigidos en normas de vida por la ideología neoliberal. Es una ideología disfrazada de ciencia. La inestabilidad y la incertitud del momento actual se deben al hecho de no haber elaborado una definición de un nuevo tipo de organización y de no haber previsto las consecuencias

\footnotetext{
${ }^{1}$ Aquí en ese trabajo consideramos organizaciones del tercer sector aquellas que no forman parte del Estado ni del mundo empresarial. Se conforman por iniciativa de personas o entes particulares, con una dimensión pública en cuanto a sus actividades que se alinean en torno a los servicios comunitarios (González Bombal, 1995: 65)
}

de la emergencia de las nuevas condiciones del mercado de trabajo. La necesidad de una reflexión y análisis sociológico sobre las diferentes realidades que traduzca, de un lado, las iniciativas innovadoras para la superación de la crisis y, por otro, la desestructuración de una realidad conocida expresándose, principalmente, a través de fenómenos como el desempleo masivo, el autoempleo, la emergencia de ocupaciones atípicas, la precarización de las condiciones de trabajo, entre otros fenómenos que se unen a las ya tradicionales formas de exclusión social.

Por eso, analizar el papel de las organizaciones del sector social y cooperativo en un contexto de incertidumbre económico-financiera y de ambigüedad social, sin reducir o simplificar la realidad que estas organizaciones enfrentan es pertinente porque no reflejan solo una actividad teórica de los investigadores académicos sino que constituye un campo de acción que se refiere a la capacidad y al pragmatismo de los actores sociales en resolver los problemas reales y cotidianos a los que se enfrentan. Paradójicamente, por más que se sepa que las relaciones sociales no son auto-explicables, sino pluricausales, los estudios o investigaciones académicas tienden a considerarlos desde una perspectiva normativa que no siempre garantiza los propósitos de una metodología de trabajo autónoma y que conduce a los investigadores a una condición de consumidores pasivos de determinadas teorías. Abordar la necesidad de una pedagogía solidaria para contrarrestar la ambigüedad y la incertidumbre económica puede ser el disparador de una hermenéutica colectiva que es necesaria (y al mismo tiempo indispensable) al permitir otras miradas, nuevas combinaciones (de conocimiento) y una mejor comprensión de los procesos colectivos en sus diferentes realidades, dado que sus dinámicas traducen un saber, una potencia transformadora que provoca nuevos agrupamientos de fuerzas sociales y apuntalan una nueva percepción del mundo.

En ese sentido, la propuesta política de la economía social y solidaria (autogestión y solidaridad) se muestra como una herramienta capaz de romper con lógicas de exploración propuestas entre capital y trabajo, porque se percibe en ella una forma de lucha directa contra la exclusión social que puede señalar otro modo de vivir, incluso, de producir la riqueza. Como efecto del cuadro de globalización de la economía y la transformación de los procesos de producción, se legitima un discurso neoliberal que, centrándose sobre principios de liberalización a ultranza, apertura comercial internacional, flexibilidad y privatización de los sistemas productivos nacionales, se presenta como explicación del cambio social y como símbolo de modernidad. Al mismo tiempo, las estrategias de desarrollo se presentan muy parecidas: flexibilidad interna y externa de las empresas, modos de organización del trabajo donde la polivalencia obrera y/o funcional aparece como señal de eficiencia empresarial que aniquila los antiguos puntos de referencia.

Los factores señalizadores de innovaciones sociales, (así como de nuevos "significados" del concepto de autogestión) no siempre se presentan como innovadores, porque no hay ruptura en los procesos sociales. Nuestro cuidado con los sentidos o significados del cambio social no solo es una preocupación semántica, frente a los dilemas sociales, independiente del tipo de proceso productivo, las nuevas formas de organi- 
zación del trabajo son redundantes y reproducen formas de actuar antiguas: vino viejo en botella nueva. Nuestra intención en este texto es identificar los límites y las posibilidades de la solidaridad propuesta por el cooperativismo que se presenta (en el siglo XXI) como una práctica social innovadora. Pensar la solidaridad como alternativa diferenciada y, principalmente, como una herramienta organizadora de la acción colectiva en los procesos productivos es fundamental cuando se tiene presente un escenario económico globalizante, principalmente en el Mercosur, porque esta lógica económica puede permitir o no el propósito de articular cadenas productivas asociativas.

La solidaridad como forma de organización del proceso de trabajo tiene una aspiración a la integralidad social, cuya pretensión de equidad se evidencia no solamente ahora, sino que ya pusieron de manifiesto los socialistas utópicos y los pioneros del cooperativismo como: Saint Simon, Charles Fourier, Luis Blanc, Pierre Joseph Proudhon y Robert Owen (Santos Rodríguez, 2005). Este último teórico señalaba:

"El objetivo primordial y necesario de toda una existencia debe ser la felicidad, pero la felicidad no se puede obtener individualmente; es inútil esperar la felicidad para uno mismo; todos debemos compartirla o en el caso contrario la mayoría de las personas nunca podrán gozarla".

Es necesario tener presente que ese deseo de equidad social no significa solamente un ejercicio lógico-intelectual, visible en la racionalidad instrumental que define objetivos y metas de un modo de acción organizacional, sino de una acción colectiva cuya intencionalidad se concreta en el ejercicio político que propone nuevas prácticas sociales de redistribución de poder entre los diferentes actores sociales. Entendemos que discutir la solidaridad en la economía social y solidaria tiene un doble sentido: primero, potenciar y maximizar la racionalidad de los actores sociales y, segundo, articular dos tipos de saberes: el conocimiento técnico-científico (de la administración) con los saberes que las personas tienen (o los saberes de la comunidad); tarea que resulta compleja y que no es frecuente realizarla. Para situar adecuadamente el problema de la articulación de esos dos tipos de saberes en la promoción de la solidaridad cooperativa es necesario otra mirada, menos abstracta y universal sobre la pedagogía socialaquí entendida como formas ${ }^{2}$ que se explicitan y se reconstruyen en la praxis colectiva.

Si la solidaridad como praxis colectiva no produce una acción efectiva y modificadora de la realidad, entonces es solamente una concepción, cuyo producto es una acción puntual, más o menos emotiva, sin capacidad de transformación cualitativa de la realidad, por ser puro ejercicio organizativo "más o menos" eficiente. Se debe tener presente que la función mani-

\footnotetext{
${ }^{2}$ Pedagogía aquí está presentada en dos sentidos: el primero, en el sentido estricto de disciplina que posibilita comprender los mecanismos existentes en las interacciones recurrentes (clases, escuela, aprendizajes) y cuáles son las acciones que predominan y cuáles son las que constituyen el otro como persona legitima. El segundo, más amplio, como formas que permiten la construcción de escenarios hipotéticos que focalizan procesos causales y situaciones que precisan ser decididas. Se trata de procedimientos trazados hoy y que tienen vinculación con situaciones futuras: auxilian en la selección de líneas de acción.
}

fiesta de los presupuestos de un modelo dado funciona como regulador de un orden social y, como tal, es selectivo y provoca redefiniciones, olvidos o negaciones. Por eso, no nos resulta extraño que los múltiples significados de la solidaridad sean aceptados, en la medida que se presentan como dispositivos técnicos y políticos capaces de enfrentar las frecuentes situaciones de incertidumbre existencial que acosan a los grupos sociales. Solidaridad es uno de esos significados que encanta, porque instaura una visión de historia colectiva como ruptura y discontinuidad, construyéndose en una serie de mutaciones inaugurales adonde no hay lugar para un proyecto divino.

"[...] cada trabajador participará de las ganancias y pérdidas del establecimiento, y tendrá voz deliberativa en su administración" (Proudhon, 1932 apud Almeida, 1983) y "adonde no puede haber separación entre trabajo y capital" (Singer, 2005).

Dicho de otro modo, la solidaridad atrae porque rompe con la lógica del capital y se evidencia como una "representación" en la cual el individuo expresa su interpelación a las condiciones reales de existencia; tiene que ver con el sentido de negación del experimentado, de un saber de los individuos o grupos que redefine determinados conceptos de gestión. Por otro lado, la autogestión en los espacios asociativos saca los conceptos de gestión del dogmatismo, del carácter unilateral, de la esclerosis, del fanatismo y del espíritu categórico, de los elementos de intimidación, de la ingenuidad y de las ilusiones de una fijación sobre un solo plano: del concepto de gestión como apenas un ejercicio técnico especializado. Siendo así, pasa a ser fundamental identificar el proceso de producción de los significados que nunca es dado por aquél que habla, sino determinado por relaciones de fuerza de las que emergen significados o efectos que pueden ser totalmente contradictorios.

\section{Los significados y sus dimensiones}

La autogestión se presenta como un conjunto de prácticas sociales datadas y localizadas históricamente (el mutualismo de Proudhon, las cooperativas de Owen, las fábricas autogestionadas en la Yugoslavia de Tito, los kibutz israelíes, etc.) que proponen la autonomía del "nosotros", cualificando la cooperación entre las personas, porque fundamenta esas prácticas en la reciprocidad, en la confianza, en la pluralidad y en el respeto al otro. Es una perspectiva democrática, donde la calidad y el carácter asociativo que se evidencia alrededor de ciertas operaciones realizadas entre personas y/o grupos explicita un modo de ejercitar la ciudadanía, independiente de los resultados alcanzados o del modo como los elementos están agrupados o asociados, constituyéndose, así, desde una perspectiva plenamente democrática. De ese modo la autogestión implica: 
a) Una modalidad de acción colectiva que resulta no solamente de una experiencia concreta, sino de la "experimentación", del vivir de otro modo.

b) Un conjunto de acciones realizadas por personas (o grupos) que están movilizadas a partir de proyectos comunes, que buscan superar las dificultades que se les presentan y que no sería posible resolverlas individualmente. Son acciones que buscan atender al imperativo categórico de construir criterios capaces de cualificar la acción colectiva a través del establecimiento de normas cuyo fundamento moral y ético considera al "otro" cómo individuo autónomo y capaz de tomar decisiones.

En ese sentido, el asociacionismo autogestionario, propuesto por la economía social y solidaria es mucho más que una simple celebración de un contrato mutuo que establece obligaciones visando objetivos comunes. La esencia de esta sociedad civil está fundada en el modo de decidir, en la unión de esfuerzos y en el establecimiento de otro tipo de acción colectiva que tiene en la reciprocidad y en el respeto al otro la implementación de otro tipo de acción social.

Referida a la vida cotidiana de las personas es posible identificar tres dimensiones esenciales de una pedagogía social. La primera, recusa la lógica economicista que reduce el quehacer humano a la búsqueda racional del interés propio y a la práctica cooperativa que tiene como objetivo final la ganancia máxima y/o al consumo compulsivo como valor por encima de cualquier otro valor particular cuando se trata de la formulación de leyes y derechos colectivos. Esto rompe con la concepción de que la cooperación solo es posible desde una perspectiva contractualista y que el interés en asociarse es resultado de motivos egoístas y narcisistas. La segunda, señala que la lógica social puede ser hegemónica, pero no es homogénea y que hay pensar la cooperación como un espacio social plural y no instrumentalizable. Finalmente, señala respeto a la ética, o sea, la interdependencia existente en cada acción individual, que cooperar es un proceso frágil, lo que puede dar lugar a fallos independientemente de los propósitos de partida.

En esta línea, una pedagogía de la precariedad emerge como la posibilidad que podría alejarnos de estas magras ganancias propuestas por la cooperación capitalista y conducirnos a opciones que apunten para un mundo más autónomo y con menos divisiones.

4. La praxis pedagógica de la solidaridad y sus diferentes implicaciones

Entiendo que la praxis pedagógica es un proceso de adquisición de saberes, de adaptación, de acomodación a una circunstancia diferente de aquella en que la persona - niño, adulto, joven, anciano - se encontraba originalmente. Deviene de una cierta historia de interacciones recurrentes y busca un modo de ser diferente de la situación original. Desde el punto de vista microsocial esta lógica consiste en considerar que los acuerdos y la capacidad de formar y dirigir las actividades de los grupos deriva de la necesidad de los valores que sustentan los grupos. Desde el punto de vista macrosocial, consiste en un conjunto de arreglos políticos a partir de los cuales se consolidan las ideas de: soberanía, representación, regla de la mayoría y sufragio universal. Dicho de otro modo, exige la liberación de las potencialidades de los miembros de un grupo en acuerdo con los intereses que son comunes y de los acuerdos que se sostienen en un proyecto comunitario, en interacciones repetitivas, o sea, en una pedagogía política. Siendo así, solamente cuando partimos de una comunidad que se pretende autónoma es que comprendemos que la actividad asociada es una condición de la creación de la comunidad. Creación que se hace como proceso auto-modelador y del mundo de relaciones en el que la afirmación de los deseos, de su capacidad imaginativa de reinventar la vida, significa retomarla desde la afirmación de su alteridad distintiva.

Es necesario tener presente los diferentes tipos de cooperación, porque en cuanto proceso ella se origina de dos movimientos contradictorios: uno narcisista y egoísta y otro basado en la gratuidad y en la reciprocidad.

La cooperación tipo 1 -instrumentalizante o narcisista-favorece y abre posibilidades para un tipo de participación en la cual los integrantes se instrumentalizan mutuamente. Ese tipo de participación es frágil y pautada por la lógica del mercado, en la que las partes cooperantes y asociadas buscan ampliar sus limitaciones a partir de la cooperación, por ejemplo: la cooperación entre un ciego/paralítico. Ese tipo de asociacionismo instrumentalizante tiene como premisa que todos somos egoístas y racionales a punto, incluso, de posibilitar situaciones en que se finge que cooperamos.

La cooperación de tipo 2 -cualificada o recíproca- en cuanto relación que depende de una esfera de actividades no tan nítidamente marcada por factores económicos tiende a ser más compleja y por eso más frágil, porque debe ser cualificada; basada en la reciprocidad, en la confianza, en la pluralidad y en el respecto al otro, dado que propone fundamentalmente la autonomía del colectivo.

Esos dos tipos de cooperación que en cierto modo son los elementos constitutivos del asociativismo fueron presentados como un artificio lógico/conceptual para buscar una tipología reduccionista, y no como elementos concretos de resignificación de una pedagogía que necesita otra mirada. Para pensar una praxis pedagógica de la precariedad es necesario tener presente algunos elementos de la lógica vigente como:

a) Considerar que vivimos en una era de potencial abundancia gracias a la tecnología y no tener en cuenta que la base material -la naturaleza- tiene sus límites.

b) La acumulación propuesta por el capital generadora de desigualdades.

c) Entender que la acumulación creciente y la producción en escala es el único recurso para evitar la inseguridad.

d) Pensar que las experiencias de cooperación son ejercicios sociales experimentales y que los cambios sociales son hechos realizados por iniciativas individuales. 
En esta situación la pedagogía de la solidaridad propone que la organización de las personas, que su unión sea mucho más que una propuesta relacionada a la solución de necesidades comunes y al alcance de mejores condiciones de vida, que sea un espacio de resistencia cuyo resultado esperado es la autonomía y la posibilidad de continuidad del grupo. En ese sentido, la pedagogía de la solidaridad proyecta un modelo de regulación alternativo en la medida que se fundamenta en el ejercicio de la ciudadanía que busca proponer modos plurales de actuación e instaurar un movimiento de interacción de los espacios económicos, sociopolíticos y culturales en una perspectiva más amplia.

Para nosotros, entretanto, a pesar de que el término autogestión se haya tornado compatible con la globalización de las técnicas de administración, su propuesta pedagógica todavía permanece bastante imprecisa. La mayoría de las veces aparece asociada a la comprensión de los procesos productivos (industriales), que se desenvolvieron asociados a determinados tipos de tecnología. De una manera general, podemos decir que el término "autogestión" está siendo utilizado mucho más para designar una matriz de conocimientos relacionada a determinados métodos o técnicas administrativas que, al mistificar el lado gerencial del proceso de trabajo, desconsideran la realidad en cuanto resultado de las prácticas sociales de actores en un determinado contexto (histórico, geopolítico, espacial, etc.) ${ }^{3}$. Por otro lado, pensar la "autogestión" a partir del referencial "cooperativo"4 significa reducirlo a la "mitología del buen salvaje" ${ }^{\prime \prime}$, que disimula la realidad social y la complejidad del proceso de producción, induciendo la creencia nostálgica de que una relación simple y sana entre aquellos que producen puede ser promovida a través de sistemas de participación y de responsabilidad escasamente gerencial.

La democratización de los espacios de trabajo no se limita a la integración por parte del trabajador de las metas y objetivos de la empresa, porque ésta exige la redefinición de las formas de participación en el proceso de trabajo. Tampoco la democratización en el trabajo se reduce a la participación individualizada y formal. Es preciso que los mecanismos de representatividad y participación no se restrinjan, en el trabajo al espacio sindical para que exista la posibilidad de participación concreta en la gestión. La construcción de una "pedagogía de la solidaridad" pasa necesariamente por la discusión, por la explicitación de las diferencias, por el conflicto. Es necesario el conflicto, entrar en una discusión implica la aceptación de las credenciales de nuestro interlocutor, así como una promesa de atenernos a las reglas y estándares del discurso. Aceptar debatir y acordar negociar los términos de la verdad suelen ser considerados señales de debilidad, circunstancias que hace

\footnotetext{
3 Esa otra lógica social necesita ser explicitada y entendida, porque se torna cada vez más común hablar de formas de cooperación capitalistas en oposición a las propuestas asociativas.

${ }^{4}$ El concepto de autogestión es un asunto que acompaña la historia del cooperativismo y todavía es tema nebuloso al punto de merecer contínuas reflexiones dada la posibilidad de implicaciones falaciosas y erróneas sobre el tema.

5 Ëliade, Mircea "muthes, rêves et mystéres. Folio/essais no* 128 Gallimard El mito del buen salvaje traduce interpretaciones apologéticas de una realidad social donde la libertad, la divisón del trabajo y los frutos del trabajo se dan de forma justa donde el hombre vive en perfecta armonía com la naturaleza.
}

que el más fuerte (o quien desee demostrar su superioridad) se muestre aún más renuente a abandonar su negativa a todo diálogo. Disputar no se refiere al significado primario de autoafirmación y de probar que los interlocutores están equivocados, al revés, es probar que los interlocutores pueden llegar a una "comunicación" a una "simetría de ambos" autenticando la igualdad y la paridad entre actores sociales.

Entendemos la "pedagogía de la solidaridad" como una construcción social, una praxis colectiva capaz de engendrar acciones y resultados aceptables para todos los individuos y grupos que producen de forma colectiva, porque se definen sobre la base de sistemas de saberes plurales cuyos valores, principios y prácticas privilegian los saberes potenciales del grupo o comunidad y la prerrogativa de las decisiones. Se presenta como resultado de una construcción colectiva que pasa por el poder de opinar y de decidir, que es compartido por todos los segmentos, de forma que garantice el equilibrio de fuerzas(multiproporcionalidad) y el respeto a los diferentes papeles de cada uno dentro del grupo o comunidad.

\section{Consideraciones precarias y no finales}

Es cierto que todo punto de vista depende de ciertas suposiciones referentes a la naturaleza de la realidad. Si eso sucede, las suposiciones funcionan como hipótesis; si eso es olvidado, funcionan como creencias y paradigmas de acción. Los paradigmas se tornan normativos, se convierten en marcos de referencia y filtros conceptuales que condicionan la manera de ver las cosas. Pensar una pedagogía de la solidaridad tiene sentido, porque su propuesta, su discurso está anclado rigurosa y esperanzadamente en el diálogo, que es que un discurso inseguro de su propia verdad y eso es el ingrediente principal constitutivo de su praxis, y la principal herramienta del proceso autogestionario. Para hacerla, así como en los procesos autogestionarios, se debe evitar la banalización de los principios de la participación y del compromiso, además es necesario que se concreten en un conjunto de medidas políticas que buscan la democratización y socializar los saberes. Esa participación supone una pedagogía política que es al mismo tiempo una política pedagógica que tiene en la Autogestión las condiciones para seguir adelante. El énfasis en el carácter de interrelación y contextual de los procesos sociales permite reconocer la complejidad en que se entretejen los múltiples sujetos sociales, las diferentes perspectivas epistemológicas y las políticas, diversas prácticas y variados contextos sociales.

Para finalizar, esta reflexión no tiene pretensión de ser exhaustiva o conclusiva, sino que se trata de realizar una aproximación introductoria e indicativa que las propuestas pedagógicas de la economía social y solidaria pueden contrarrestar la lógica del mercado de contaminar los proyectos de educación cuando se piensa la economía social y solidaria. Se trata de una formulación precaria porque el ejercicio analítico consiste 
meramente en la compresión teórica de que no puede haber una única verdad en el mundo asociativo. Y, además, que la verdad solo puede emerger al final de una conversación, y a partir de una conversación genuina, donde ninguno de los interlocutores sabe o pude saber si su validez está dada, porque el concepto de verdad es eminentemente agonístico. Nace del enfrentamiento de ideas, siempre renuentes a ceder. Por eso ahora es vuestro turno como lectores de seguir adelante con la reflexión.
Bibliografía

ARENDT, Hanna (1999): A condição Humana, 9 edição. Rio de Janeiro, Editora Forense Universitária.

BACHELARD, G. (1985): O novo espírito cientifico. Rio de Janeiro, Ed. Tempo brasileiro.

CASTELLS, M. (1991): O poder da identidade. São Paulo, Ed. Paz e Terra.

CASTELLS, M. (1999): A sociedade em rede. São Paulo, Paz e Terra.

CASTEL, ROBERT (2001): A questão social, 5 edição. São Paulo, Ed. Movimento. DEWEY, John (2008): “Em busca da grande democracia 1927. Escritos Políticos Escolhidos", en Augusto De Franco e Thamy Pogrebinschi. Porto Alegre, EDIPUCS

GIDDENS, A. (2000): Mundo em descontrole. O que a globalização está fazendo por nós. São Paulo, Ed. Record.

HONNETH, AXEL (2004): Luta por reconhecimento - A gramática moral dos Conflitos Sociais, São Paulo, ed. Record.

RIFKIN, Jeremi (1999): O século da biotecnologia. São Paulo, Makron books BHABHA, H. (1998): O local da cultura. Belo Horizonte, UFMG.

DAYRELL, J.T. (2002): O jovem como sujeito social (GT03), en Reunião Anual da ANPED, 25, Caxambú. Anais... Caxambú, ANPED. Disponible en: <http:// www.anped.org.br/25/juarezdayrellt03.rtf> Acceso en: 27 maio 2018.

FLEURI, R.M.; BITENCOURT, S.M. y SCHUCMAN, L.V. (2002): "A questão da diferença na educação: para além da diversidade", en Reunião Anual da ANPED, 25, Caxambú. Anais... Caxambú, ANPED. Disponible en: <http:// www.anped.org.br/25/sessoesespeciais/reinaldofleuri.doc> Acceso en: 27 maio 2018.

LARROSA, J. y LARA, N. P. (orgs.) (1998): Imagens do outro. Petrópolis, Vozes. PIERUCCI, A.F. (2002): “Ciladas da diferença. São Paulo: Editora 34, 1999ROSS, P.R. Estado e educação: implicações do liberalismo sobre a constituição da educação especial e inclusiva (GT15)", en Reunião Anual da ANPED, 25, Caxambú. Anais... Caxambú, ANPED, 2002. Disponible en: http://www. anped.org.br/25/pauloricardorosst15.doc. Acceso en: 27 maio 2018.

SILVA, G.F. (2002): "Interculturalidade e educação de jovens: processos identitários no espaço urbano urbano popular (GT06)", en Reunião Anual da ANPED, 25, Caxambú. Anais... Caxambú, ANPED. Disponible en: http:// www.anped.org.br/25/gilbertoferreirasilvat06.rtf. Acceso en: 27 maio 2018.

VANISTENDAEL, S. (1995): Como crecer superando los percances; Resiliência: capitalizar las fuerzas del individuo. Ginebra, Oficina Internacional Católica de la Infancia (BICE). 